\title{
Anemia in Glucose Transporter Type 1 Deficiency Syndrome: Often Expected, Rarely Encountered, and with a Fascinating Explanation*
}

\author{
Michèl A. Willemsen ${ }^{1}$ \\ ${ }^{1}$ Department of Pediatric Neurology, Donders Institute for Brain, \\ Cognition and Behaviour, Radboud University Medical Center, \\ Nijmegen, The Netherlands \\ Neuropediatrics 2017;48:327-328.
}

"Why don't patients with glucose transporter type 1 deficiency syndrome (GLUT1DS) suffer from anemia if the protein is so important for erythrocytes?" This single question is undoubtedly among the students' favorites at teaching courses on GLUT1DS during the past 15 years or so. Since I'm not aware of any study on the life span of red blood cells of patients with GLUT1DS in general, and since I have never encountered anemia as a clinical problem in these patients, I simply answer the students that anemia is not a common feature of GLUT1DS. Very often this leads to some disappointment since they feel that my statement undermines the lesson that erythrocyte glucose uptake studies can be used to functionally prove the deleterious effect of SLC2A1 mutations in the laboratory. Those who love exceptions to the rule and persist will recall some extremely rare patients with GLUT1DS and hemolytic anemia. Indeed, anemia in these patients is caused by the SLC2A1 mutation; however, it is not the consequence of a defective glucose transport.

From the patient described in this issue of Neuropediatrics ${ }^{1}$ and those in three earlier reports, ${ }^{2-4}$ we can learn that certain SLC2A1 mutations lead to the synthesis of an abnormal GLUT1 protein that harbors two (instead of one) principal functional defects. First, the abnormal protein impedes glucose transport into the brain (and into erythrocytes), as expected. Second, the abnormal protein leaks cations due to a change of its tertiary structure that widens its pore region. The former abnormality causes the neurologic disorder that we know as GLUT1DS, in its classic or milder form, and with its wonderful response to treatment with the ketogenic diet. Altered intracellular

\footnotetext{
* This article is an editorial comment on "Another case of glucose transporter 1 deficiency syndrome with periventricular calcification, cataracts, hemolysis, and pseudohyperkalemia" by Shibata et al (Neuropediatrics 2017; doi: 10.1055/s-0037-1603520).
}

concentrations of sodium, potassium, and calcium, but not glucose shortage of red blood cells, cause hemolytic anemia. In patients with such SLC2A1 mutations, the cation leak may aggravate the neurologic consequences of glucose shortage and may cause periventricular calcifications. In the lens, cation leakage may lead to cataracts.

In 2008, Weber et al were the first to report on anemia in the context of GLUT1DS. ${ }^{2}$ They described a family with a relatively mild neurologic disorder, dominated by paroxysmal exertioninduced dyskinesia, and hemolytic anemia. The affected members harbored an in-frame 12-bp deletion (c.1022_1033del) of SLC2A1, predicting the loss of four amino acids (p.Q282_S285del) within the pore region of GLUT1. Additionally, they linked the GLUT1 protein change to the leakage of cations from erythrocytes. The second and third reports on this topic appeared in 2011 and 2012, respectively, together describing three patients suffering from a severe, multisystem disorder, resembling the present case. ${ }^{3,4}$ In one patient, the SLC2A1 mutation led to p.Gly286Asp, neighboring the protein change found by Weber et al. The other two patients carried the same mutation as the present case, resulting in the loss of isoleucine at either position 435 or 436 of GLUT1.

Shibata et al illustrate the fact that GLUT1DS is characterized by a typical cerebrospinal fluid (CSF) profile with low glucose and low lactate concentrations, as recently described. ${ }^{5}$ Importantly, the report emphasizes the fact that such a CSF profile should raise the suspicion of GLUT1DS, also in a patient with a neurologic disorder that does not resemble the classic phenotype. An important reason for the latter statement is that, as far as I am aware, there are no other disorders known with the CSF profile of GLUT1DS. Furthermore, GLUT1DS is a treatable disorder; thus, every meaningful clue to its diagnosis should be taken seriously.

(c) 2017 Georg Thieme Verlag KG Stuttgart · New York
DOI https://doi.org/ 10.1055/s-0037-1603519. ISSN 0174-304X.
Address for correspondence Michèl A. Willemsen, MD, PhD, Department of Pediatric Neurology, Donders Institute for Brain, Cognition and Behaviour, Radboud University Medical Center, PO Box 9101, 6500 HB Nijmegen, The Netherlands (e-mail: michel. willemsen@radboudumc.nl). 
In short, certain SLC2A1 mutations not only disturb the glucose transport capacity of GLUT1 but also cause leakage of cations through the cell membrane through the abnormally shaped protein. Patients with such mutations are the only cases with (classic or mild) GLUT1DS in whom anemia is a feature of the disorder. The phenotype of patients with classic GLUT1DS and anemia may at first glance resemble complex multisystem disorders, for example, congenital infections or pseudo-TORCH syndrome, and as a consequence, the clinical suspicion of GLUT1DS may be low or even absent. Fortunately, GLUT1DS can easily be recognized based on its unique and very simple biomarker profile in CSF. Bearing in mind the discussions with my students, I finally want to emphasize the importance of case reports. We neither would have known that anemia indeed may occur in GLUT1DS nor would have we learned about an intriguing and unexpected disease mechanism if these cases of individuals and families had not been published.

\section{References}

1 Shibata T, Kobayashi K, Yoshinaga H, Ono H, Shinpo M, KagitaniShimono K. Another case of GLUT1 deficiency syndrome with periventricular calcification, cataracts, hemolysis, and pseudohyperkalemia. Neuropediatrics 2017; doi: 10.1055/s-0037-1603520

2 Weber YG, Storch A, Wuttke TV, et al. GLUT1 mutations are a cause of paroxysmal exertion-induced dyskinesias and induce hemolytic anemia by a cation leak. J Clin Invest 2008;118(06):2157-2168

3 Flatt JF, Guizouarn H, Burton NM, et al. Stomatin-deficient cryohydrocytosis results from mutations in SLC2A1: a novel form of GLUT1 deficiency syndrome. Blood 2011;118(19):5267-5277

4 Bawazir WM, Gevers EF, Flatt JF, et al. An infant with pseudohyperkalemia, hemolysis, and seizures: cation-leaky GLUT1-deficiency syndrome due to a SLC2A1 mutation. J Clin Endocrinol Metab 2012;97(06):E987-E993

5 Leen WG, Wevers RA, Kamsteeg EJ, Scheffer H, Verbeek MM, Willemsen MA. Cerebrospinal fluid analysis in the workup of GLUT1 deficiency syndrome: a systematic review. JAMA Neurol 2013;70(11):1440-1444 